\title{
Equivalence of Dirac and Intrinsic Quantization for Non-free Group Actions
}

\author{
C. Emmrich \\ Fakultät für Physik der Universität Freiburg, Hermann-Herder-Str. 3 D-7800 Freiburg, FRG
}

Received April 2, 1992

\begin{abstract}
In this article we generalize some results on the equivalence of Dirac quantization and intrinsic quantization proven in [3]: We consider systems with first class constraints that may be considered as the vanishing of the momentum map to a lifted group action, but drop the assumption that the group action is free as well as the assumption that the group is compact. Using a generalized Weyl ordering prescription applicable to arbitrary cotangent bundles we derive necessary and sufficient conditions for the equivalence of the two approaches for different classes of functions analogous to those for the free case, although the proofs given in [3] must be considerably modified and refined due to the noncompactness of the orbits and the lack of sufficiently many invariant vector fields. The same strong obstruction as in the free case is found if one requires equivalence for all invariant functions, essentially only admitting trivial bundles.
\end{abstract}

\section{Introduction}

The different approaches to the quantization of systems with first class constraints, namely extrinsic (in particular Dirac) quantization, which first quantizes the unconstrained system and then imposes the constraints as conditions on the states, and intrinsic quantization, which first classically eliminates the constraints and then quantizes the resulting unconstrained system, are known not to be equivalent in general. However, for the case that the constraints consist in the vanishing of the canonical momentum map belonging to the lift of a group action on configuration space, some general positive results have been proven in the literature concerning the existence of a natural isomorphism between the Hilbert spaces which intertwines the operators corresponding to observables at most linear in the momenta ([4-6,3] and references cited therein).

In [3] we have proven for the case of the free action of a compact group that for a reasonable quantization scheme this natural isomorphism of Hilbert spaces intertwines the operators corresponding to observables of higher order in the momenta only under additional, rather restrictive conditions. In these proofs the 
existence of sufficiently many invariant polynomials and of sufficiently many invariant vector fields was extensively used.

In the case of a non-free action there are more restrictions on invariant polynomials than in the free case: As the isotropy group of a point acts on the tangent space of the extrinsic configuration space $E$ at that point in a nontrivial way, the coefficients $T^{\mu_{1} \ldots \mu_{k}}(q)$ of the polynomials $T^{\mu_{1} \ldots \mu_{k}}(q) p_{\mu_{1}} \ldots p_{\mu_{k}}$ at the fixed point $q$ are not independent but have to be invariant under the isotropy group of the respective point. Furthermore, the proof for free group actions given in [3] crucially depends on the compactness of $\mathbf{G}$ and the existence of sufficiently many invariant vector fields, which allow to write any invariant polynomial as a linear combination of polynomials of the form $\left(X_{1} \vee \ldots \vee X_{k}\right)^{\mu_{1} \ldots \mu_{k}} p_{\mu_{1}} \ldots p_{\mu_{k}}$ for invariant vector fields $X_{i}$. In the case of a non-free action this is no longer possible: In the extreme case that the normalizer of the typical isotropy group $\mathbf{I}$ in $\mathbf{G}$ equals I there are no invariant vertical vector fields at all, whereas the set of invariant vertical polynomials certainly is nonzero.

Hence, one might hope that under favourable circumstances the requirement of the Dirac equivalence condition for invariant polynomials might be less restrictive in the non-free case than in the free case, as the class of functions which have to fulfill the equivalence condition is subject to more restrictions.

In this article we shall show, however, that the same restrictive theorems as for free actions hold. In particular, if the Dirac equivalence condition shall hold for arbitrary invariant polynomials, the extended configuration space has to be a trivial bundle over the reduced configuration space, provided the latter is simply connected.

In more detail, the situation we are going to examine is the following: Let an arbitrary Lie group $\mathbf{G}$ with Lie algebra $\mathscr{G}$ act properly on a manifold $E$ such that all isotropy groups are conjugate to a fixed subgroup I. (This means no severe restriction, since it is known that for a proper group action there is an open dense subset of $E$ of points fulfilling that condition. All operators we are going to study are local differential operators and we shall only be concerned with formal selfadjointness anyway. Giving up the properness of the action would imply that the reduced configuration space is not even Hausdorff.) $E$ will play the role of the extrinsic configuration space. We assume that the system is subject to the first class constraints $\mathscr{J}_{A}=0 \forall A \in \mathscr{G}$, where $\mathscr{J}$ denotes the canonical momentum map to the lifted action of $\mathbf{G}$ on $T^{*} E$. The reduced phase space is $\mathscr{J}^{-1}(0) / \mathbf{G}$ which under the conditions above is canonically isomorphic to $T^{*} Q$ with $Q=E / \mathbf{G}$. Hence, we may consider $Q$ as the reduced configuration space.

In the following section we shall briefly review the structure of the spaces we are considering and the G-invariant metrics on them. In Sect. 3 we shall shortly summarize the results of [5] on the existence of a natural isomorphism and sketch the quantization method we are going to use for the quantization of arbitrary observables. This quantization is the modification of the generalized Weyl ordering prescription of [7], used in [3].

In the last section we are going to prove the equivalence theorems. It will be shown that even with the strongest possible restriction on the set of admissible functions on $T^{*} E$, which singles out a unique representative corresponding to an observable on $T^{*} Q$, the "orbit volume" generally has to be constant in order for the equivalence to hold for all quadratic observables. This in particular forbids the possibility of getting a formal equivalence in the case that the isotropy groups are not all conjugate, since in that case the orbit volume never is constant. 
With weaker restrictions on the set of admissible functions on $T^{*} E$ even much more restrictive necessary and sufficient conditions for the equivalence will follow. If one admits all invariant functions on $T^{*} E$ as representatives of observables on $T^{*} Q$, the extended configuration space has to be a trivial bundle over $Q$, provided $Q$ is simply connected.

These negative results are of particular importance, since even more elaborate extrinsic quantization schemes like BRST quantization are under certain technical assumptions for globally G-invariant functions equivalent to Dirac quantization.

\section{The Structure of Simple G-Spaces}

In this section we summarize some well known facts about the structure of a manifold $E$ on which a Lie group $\mathbf{G}$ acts properly such that all isotropy groups are conjugate to a fixed subgroup $\mathbf{I} \subset \mathbf{G}[2]$ :

Due to the properness of the $\mathbf{G}$-action, $\mathbf{I}$ is compact. Let $\mathbf{N}$ denote the normalizer of $\mathbf{I}$ in $\mathbf{G}$ and $\mathbf{G}_{q}$ the isotropy group of $q \in E$. The subset

$$
P:=\left\{q \in E \mid \mathbf{G}_{q}=\mathbf{I}\right\}
$$

is a submanifold of $E$ which has the structure of a left $\mathbf{N} / \mathbf{I}$-principal bundle over $Q:=E / \mathbf{G}$. The manifold $E$ is an associated bundle with fiber $\mathbf{G} / \mathbf{I}$ on which $\mathbf{N} / \mathbf{I}$ acts from the right. We denote the bundle projection by $\pi: E \rightarrow Q$ and by $\mathscr{G}, \mathscr{N}, \mathscr{I}$ the Lie algebras corresponding to $\mathbf{G}, \mathbf{N}$ and $\mathbf{I}$, respectively.

There is a one-to-one correspondence between $\mathbf{G}$-invariant metrics $\mathbf{g}$ on $E$ and triples $(\tilde{\mathbf{g}}, \alpha, \mathbf{h})$, where $\tilde{\mathbf{g}}$ is a metric on $Q, \alpha$ is a connection form on $P$ as an $\mathbf{N} / \mathbf{I}$-principal bundle over $Q$, and $\mathbf{h}$ is a smooth mapping assigning to any $\tilde{q} \in Q$ an invariant Riemannian metric $\mathbf{h}(\tilde{q})$ on the orbit $\pi^{-1}(\tilde{q})$.

As in the free case, the vertical distribution on $E$ is defined by the tangent spaces to the orbits. For a given metric $\mathbf{g}$ a $\mathbf{G}$-invariant horizontal distribution on $E$ can be defined as the orthogonal complement of the tangent spaces to the orbits. For any $q \in E$ the isotropy group $\mathbf{G}_{q}$ of $q$ acts on the tangent space $T_{q} E$. In particular, for $q \in P$, I acts on $T_{q} E$ leaving the vectors tangent to $P$ invariant. For an orthogonal representation of a compact group any invariant subspace not containing any invariant vector is orthogonal to every invariant vector. Using this fact and some dimension arguments, one may show that the horizontal vectors at a point $q \in P$ defined as above are tangent to $P$. Hence, the restriction of the horizontal distribution on $E$ to $P$ defines a connection on $P$. On the other hand, given a connection and hence a horizontal distribution on $P$, there obviously is a unique $\mathbf{G}$-invariant horizontal distribution on $E$ whose restriction to $P$ coincides with the horizontal distribution on $P$.

The metric $\tilde{\mathbf{g}}$ on $Q$ may now be defined as in the free case by

$$
\tilde{\mathbf{g}}(\tilde{X}(\tilde{q}), \tilde{Y}(\tilde{q}))=\mathbf{g}\left(\tilde{X}^{h}(q), \tilde{Y}^{h}(q)\right)
$$

for some $q \in \pi^{-1}(\tilde{q})$, where $\tilde{X}^{h}$ denotes the horizontal lift of $\tilde{X} . \mathbf{h}(\tilde{q})$ is just the metric on the orbit $\pi^{-1}(\tilde{q})$ induced by $\mathbf{g}$.

For $q \in P$ we denote by $\mathscr{H}_{q}$ the horizontal space at $q$, for a vector field $X$ over $E$ by $\mathscr{H}(X)$ its horizontal projection, by $\mathscr{V}(X)$ its vertical projection. 
Now, given $(\tilde{\mathbf{g}}, \alpha, \mathbf{h})$, we may set

$$
\mathbf{g}(q)(X, X):=\tilde{\mathbf{g}}(\pi(q))\left(\pi_{*} X, \pi_{*} X\right)+\mathbf{h}(q)(\mathscr{V}(X), \mathscr{V}(X))
$$

which obviously defines a $\mathbf{G}$-invariant metric $\mathbf{g}$ on $E$.

\section{Dirac Quantization on Simple G-Spaces}

We shall first give a short summary of those results of [5] which will be needed in the following and then present the quantization scheme for arbitrary observables used in [3] for the case of free group actions:

In order to avoid dealing with half-forms instead of half-densities we assume that $E$ and $Q$ are orientable. We denote by $\Omega^{1 / 2}(E)$ the space of half-densities on $E$, by $\Omega_{\text {phys }}^{1 / 2}(E)$ the space of half-densities $\varphi$ fulfilling the Dirac condition

$$
\mathscr{2}\left(\mathscr{J}_{A}\right) \varphi=0 \quad \forall A \in \mathscr{G},
$$

by $\Omega^{1 / 2}(Q)$ the space of half-density on $Q$, and by $\mathbf{H}_{\text {int }}$ the closure of the subset of $\Omega^{1 / 2}(Q)$ consisting of square integrable half-densities, which is just the intrinsic Hilbert space. Here, any function on $T^{*} E$ of the form $f\left(\alpha_{q}\right)=\alpha_{q}\left(X_{q}\right)+g(q)$ for some vector field $X$ on $E$ and some function $g \in \mathscr{F}(Q)$ is quantized by an operator $\mathscr{Q}(f)$ defined by:

$$
\mathscr{Z}(f) \varphi:=\left(-i \mathbf{L}_{X}+\mathbf{g}\right) \varphi,
$$

where $\mathbf{L}_{X}$ denotes the Lie derivative of half-densities.

If $\mathbf{G}$ is compact we may define $\mathbf{H}_{\text {phys }}$ in an analogous way as the closure of the subset of $\Omega_{\mathrm{phys}}^{1}(E)$ consisting of square integrable half-densities, whereas the nonvanishing elements of $\Omega_{\text {phys }}^{1 / 2}(E)$ are never square integrable in the non-compact case, where $\mathscr{2}\left(\mathscr{J}_{A}\right)$ only has generalized eigenfunctions to the eigenvalue zero.

We assume that there is a nowhere vanishing half-density $s_{0}$ on $\mathbf{G} / \mathbf{I}$ that is $\mathbf{G}$-invariant from the left and $\mathbf{N}$ or, equivalently, $\mathbf{N} / \mathbf{I}$-invariant from the right. Using $s_{0}$ we may define half-forms $s(\pi(q))$ on all orbits via an arbitrary local trivialization of $P$, which induces a trivialization of $E$ and hence an identification of the orbits with $\mathbf{G} / \mathbf{I}$. Hence, we may define a smooth bijection:

$$
U: \Omega^{1 / 2}(Q) \rightarrow \Omega_{\text {phys }}^{1 / 2}(E)
$$

by

$$
(U \varphi)(q)\left(\left(V_{i}, X_{\mu}\right)\right):=s(\pi(q))\left(\left(V_{i}\right)\right) \cdot \varphi\left(\left(\pi_{*} X_{\mu}\right)\right),
$$

where $\left(V_{i}, X_{\mu}\right)$ is a frame at $q$ which consists of vertical vectors $\left(V_{i}\right)$ and arbitrary vectors $\left(X_{\mu}\right)$. For this construction the $\mathbf{N} / \mathbf{I}$-invariance from the right is needed for $s(\pi(q))$ to be well defined, independent of the choice of the trivialization, and the $\mathbf{G}$-invariance from the left is needed for the image of $U$ to fulfill the Dirac condition.

The existence of $s_{0}$ is equivalent to the condition

$$
\left|\operatorname{det} \overline{\operatorname{Ad}_{n}}\right|=1 \forall n \in \mathbf{N} \text {, }
$$


where $\overline{\mathrm{Ad}}$ denotes the representation of $\mathbf{N}$ on $\mathscr{G} / \mathscr{I}$ induced by the adjoint representation of $\mathbf{G}$ on $\mathscr{G}$ [5]. This condition implies, and for the case of connected $\mathbf{N}$ is equivalent to:

$$
\operatorname{tr} \overline{\operatorname{ad}}_{A}=0 \forall A \in \mathscr{N},
$$

which is in particular fulfilled for all compact groups and more generally for all groups admitting a biinvariant nondegenerate metric. In the following we shall always assume that condition (2) is satisfied.

In this case $U$ defines a smooth bijection which intertwines the operators corresponding to invariant observables at most linear in the momenta. Furthermore, if $\mathbf{G}$ is compact, $U$ even defines an isomorphism of the Hilbert spaces $\mathbf{H}_{\text {int }}$ and $\mathbf{H}_{\text {phys }}$ when $s_{0}$ is suitably normalized such that $\operatorname{vol}(\mathbf{G} / \mathbf{I})=1$.

Now we choose a $\mathbf{G}$-invariant metric $\mathbf{g}$ on $E$. The metrics $\mathbf{g}$ on $E$ and $\tilde{\mathbf{g}}$ on $Q$ induce canonical measures $d \mu$ and $d \tilde{\mu}$ on $E$ and $Q$, respectively, leading to canonical isomorphisms:

$$
\Omega^{1 / 2}(E) \cong \mathscr{F}^{\mathbb{C}}(E), \quad \Omega_{\text {phys }}^{1 / 2}(E) \cong\left(\mathscr{F}^{\mathbb{C}}(E)\right)_{\mathbf{G}}, \quad \Omega^{1 / 2}(Q) \cong\left(\mathscr{F}^{\mathbb{C}}(Q)\right),
$$

where $\left(\mathscr{F}^{\mathbb{C}}(E)\right)_{\mathbf{G}}$ denotes the set of $\mathbf{G}$-invariant complex smooth functions on $E$.

With these identifications, the isomorphism $U$ may be written in a simple form. To this end we define a function $\kappa$ by

$$
\kappa(\tilde{q})=\sqrt{\operatorname{det}\left(\mathbf{g}(q)\left(A_{i}^{*}(q), A_{j}^{*}(q)\right)\right)},
$$

where $q \in P \cap \pi^{-1}(\tilde{q})$ and the $A_{i}$ form an orthonormal basis of the normal space $\mathscr{M}$ to $\mathscr{I}$ in $\mathscr{G}$ with respect to some fixed $\operatorname{Ad}(\mathbf{I})$-invariant scalar product on $\mathscr{G}$ (such a scalar product always exists since $\mathbf{I}$ is compact by the properness of the action). Here, condition (2) guarantees that $\kappa$ is well defined. With this definition the isomorphism takes the same form as in the free case:

$$
U: \mathscr{F}^{\mathbb{C}}(Q) \rightarrow\left(\mathscr{F}^{\mathbb{C}}(E)\right)_{\mathrm{G}} \subset \mathscr{F}^{\mathbb{C}}(E), \quad \phi_{\text {int }} \mapsto \pi^{*}\left(\frac{\phi_{\text {int }}}{\sqrt{\kappa}}\right) .
$$

We call an open subset $D$ of $E$ cylinder-shaped if there is a local trivialization $\Phi: U \subset E \rightarrow V \times \mathbf{G} / \mathbf{I}$ and open subsets $\hat{V} \subset V \subset Q$ and $W \subset \mathbf{G} / \mathbf{I}$ such that $D=\Phi^{-1}(\hat{V} \times W)$ and the volume of $D$ is finite. With this definition one may easily show the following lemma, which gives a kind of substitute for the unitarity of $U$ in case of a non-compact group:

Lemma 1. Let $D$ be cylinder-shaped. Then there is a constant $C_{D}$ such that

$$
\langle\phi, \psi\rangle=C_{D}\left\langle\chi_{D} U \phi, \chi_{D} U \psi\right\rangle \quad \forall \phi, \psi \in \mathscr{F} \mathbb{C}(\hat{V}),
$$

where $\chi_{D}$ is the characteristic function of $D$.

For the quantization of arbitrary functions on $T^{*} E$ and $T^{*} Q$ we use the modified form of the generalized Weyl ordering [7] presented in [3] which uses functions instead of half-densities. The operators $2(f)$ corresponding to a function which is a polynomial in the momenta is defined in a coordinate independent way, yielding formally selfadjoint differential operators whose degree is the degree of the respective polynomial. 
Using coordinates $x$ on the respective configuration space $E$ or $Q$, a very explicit local expression for $\mathscr{2}(f)$ may be derived for polynomials with support in the domain of the coordinate charts: For $f=T^{i_{1} \ldots i_{m}}(x) p_{i_{1}} \ldots p_{i_{m}}$ we get

$$
\begin{aligned}
\langle\phi, \mathscr{Q}(f) \psi\rangle= & (2 i)^{-n} \int \sqrt{\operatorname{det}\left(g_{i j}(x)\right)} d x^{1} \ldots d x^{n} T^{i_{1}} \ldots i_{m} \frac{\partial^{m}}{\partial v^{i_{1}} \ldots \partial v^{i_{m}}} \\
& \times\left.\chi\left(x, v_{x}\right)\left\{\left(\exp _{x}^{*} \psi\right) \overline{\left(\left(\exp _{x} \circ I_{x}\right)^{*} \phi\right)}\right\}(x, v)\right|_{v=0}
\end{aligned}
$$

for a suitable cutoff function $\chi$ and smooth function $\phi, \psi$ of sufficiently rapid decrease. Here $I_{x}$ denotes the reflection in $T_{x} E: I_{x}(v)=-v$. As the quantization scheme maps the sum of two functions to the sum of the corresponding operators, we may restrict ourselves in the following to such functions with suitable support.

Functions which are at most linear in the momenta: $f\left(\alpha_{q}\right)=\alpha_{q}\left(X_{q}\right)+g(q)$ are quantized by

$$
\mathscr{Z}(f) \phi=\left\{-i\left(\nabla_{X}+\frac{1}{2} \operatorname{div} X\right)+g(q)\right\} \phi,
$$

which is equivalent to (1), whereas $f=T^{i j} p_{i} p_{j}$ is quantized by the operator

$$
\mathscr{Q}(f)=-\left[T^{i j} \nabla_{i} \nabla_{j}+\left(\nabla_{i} T^{i j}\right) \nabla_{j}+\frac{1}{4}\left(\nabla_{i} \nabla_{j} T^{i j}\right)\right] \text {. }
$$

\section{Equivalence Theorems}

Before proving equivalence theorems analogous to those proven in [3] for the free and compact case, we state two rather technical lemmas which will be needed in the following for the non-compact case and whose proofs may be found in the appendix:

Lemma 2. Let $\kappa$ be constant. Let $D=\Phi^{-1}(\hat{V} \times W)$ be a cylinder-shaped open subset and $X$ a $\mathbf{G}$-invariant vector field on $E$ such that the support of $\pi_{*} X$ is contained in $\hat{V}$. Then:

$$
\int_{D} \operatorname{div} X d \mu=0
$$

Lemma 3. Assume $\kappa$ is constant. Let $D$ be a cylinder-shaped open subset of $E$, and $\phi, \psi \in(\mathscr{F} \mathbb{C}(E))_{\mathrm{G}}$ with the projections of the supports of $\phi$ and $\psi$ lying in a suitable coordinate patch of $Q$. Let $\chi_{D}$ denote the characteristic function of $D, \hat{\phi}=\chi_{D} \phi$. Then formula (5) holds for the matrix element $\langle\hat{\phi}, \mathscr{Q}(f) \psi\rangle$, although $\hat{\phi}$ is not a smooth function of rapid decrease.

The last lemma is nontrivial, since, even in the case of the configuration space being $\mathbb{R}^{n}$, formula (5) generally is only valid for smooth functions of rapid decrease, as otherwise there are boundary terms when moving the derivatives acting on $\phi$ to $\psi$ via partial integration. The validity of (5) is guaranteed by the special form of $D$ and the invariance of $\phi$ and $\psi$.

With those prerequisites we may now examine the equivalence problem for arbitrary observables which are polynomial in the momenta: First, we recall that to a given observable $\tilde{f} \in \mathscr{F}\left(T^{*} Q\right)$ corresponds a whole equivalence class of functions on $T^{*} E$, elements of which differ by functions vanishing on the constraint set. The 
equivalence condition for observables is the requirement that the canonical isomorphism $U$ intertwines the operators $\mathscr{2}(\tilde{f})$ on $\mathscr{F}^{\mathbb{C}}(Q)$ and $\mathscr{Q}(f)$ on $(\mathscr{F} \mathbb{C}(E))_{\mathbf{G}}$ for suitable representatives $f \in \mathscr{F}\left(T^{*} E\right)$ of $\tilde{f}$. As explained in [3] we have to restrict the class of admissible representatives to at least those which are globally $\mathbf{G}$ invariant, but in order to get less restrictive equivalence theorems it is usually appropriate to restrict this class even more:

In [3] we called a polynomial horizontal (vertical), if it is a polynomial in the horizontal (vertical) momenta only (defined via the horizontal distribution corresponding to the connection form $\alpha$ ), and strongly admissible if it is the sum of invariant horizontal and vertical polynomials. The strongest possible restriction is that to invariant horizontal representatives, which fixes a unique representative to any $\tilde{f} \in \mathscr{F}\left(T^{*} Q\right)$. However, since those polynomials do not have any vertical part, the corresponding operators do have a very big (possibly generalized) eigenspace in $\mathscr{F}^{\mathbb{C}}(E)$ to the eigenvalue 0 . Since this is a very unpleasant feature from a practical point of view (in particular, if one wants to compute Green's functions), one is interested in admitting at least strongly admissible functions.

For the different restrictions on the admissible representatives we get the following three theorems which give necessary and sufficient conditions for the equivalence to hold for the respective classes:

Theorem 4. The Dirac equivalence condition for arbitrary "horizontal" quadratic observables holds iff the volume $\kappa$ of the orbits satisfies the equation $\tilde{\nabla} d \log (\kappa)=0$. In particular, if $Q$ is compact or the metric $\tilde{g}$ does not admit any Killing vector fields, $\kappa$ has to be constant. On the other hand, if $\kappa$ is constant, then the Dirac equivalence condition holds for arbitrary "horizontal" observables.

Proof. As any horizontal vector on $P$ is I-invariant, it has a continuation to a G-invariant horizontal vector field on $E$. Hence, the proof of the necessary condition $\tilde{\nabla} d \log (\kappa)=0$ given in [3, Theorem 6] for the free case completely goes through in the generalized case.

Now, let $\kappa$ be constant. As for free group actions, any horizontal polynomial $f$ is a linear combination of polynomials of the form $\left(X_{1} \vee \ldots \vee X_{k}\right)^{\mu_{1} \ldots \mu_{k}} p_{\mu_{1}} \ldots p_{\mu_{k}}$ for invariant horizontal vector fields $X_{i}$. This may be easily seen using a local trivialization of $E$, which induces a local embedding $i: U \subset Q \rightarrow E$ of $Q$ in $E$, and the fact that any horizontal vector field over $i(Q)$ has a unique G-invariant continuation to $E$. Hence, using the fact that for any $\varphi \in \mathscr{F}(Q)$,

$$
\left.\exp _{q}^{*}\left(\pi^{*} \varphi\right)\right|_{\mathscr{H}_{q}}=\left.\left(T_{q} \pi\right)^{*}\left(\exp _{\pi(q)}^{*} \varphi\right)\right|_{\mathscr{H}_{q}},
$$

and the constancy of $\kappa$, the proof given in [3, Theorem 4] may be easily modified for the generalized case: For any $\widetilde{\phi}, \tilde{\psi} \in \mathscr{F}^{\mathbb{C}}(Q)$ and any $a \in \mathscr{H}_{x}$, we get

$$
\left.\frac{d^{k}}{d \lambda^{k}} D(x, \lambda a)\right|_{\lambda=0}=\left.\kappa^{-1} \frac{d^{k}}{d \lambda^{k}} \tilde{D}\left(\pi(x), \lambda T_{x} \pi(a)\right)\right|_{\lambda=0},
$$

where

$$
\begin{aligned}
& D(x, v)=\chi(x, v)\left\{\left(\exp _{x}^{*} U \tilde{\psi}\right) \overline{\left.\left(\exp _{x} \circ I_{x}\right)^{*} U \tilde{\phi}\right)}\right\}(v), \\
& \tilde{D}(\tilde{x}, \tilde{v})=\tilde{\chi}(\tilde{x}, \tilde{v})\left\{\left(\exp _{\tilde{x}}^{*} \tilde{\psi}\right) \overline{\left.\left(\operatorname{(xp}_{\tilde{x}} \circ I_{\tilde{x}}\right)^{*} \tilde{\phi}\right)}\right\}(\tilde{v})
\end{aligned}
$$

for suitable cutoff functions $\chi$ on $T E$ and $\tilde{\chi}$ on $T Q$. 
As in the case of a free action, the $\mathbf{G}$-invariant function $\left(X_{1} \vee \ldots \vee X_{r}\right)^{i_{1} \ldots i_{r}}$ $\times p_{i_{1}} \ldots p_{i_{r}}$ for $\mathbf{G}$-invariant vector fields $X_{1}, \ldots, X_{r}$ on $E$ corresponds via (4) to the function $\left(\pi_{*} X_{1} \vee \ldots \vee \pi_{*} X_{r}\right)^{\mu_{1} \ldots \mu_{r}} \tilde{p}_{\mu_{1}} \ldots \tilde{p}_{\mu_{r}}$ on $T^{*} Q$, we get from (9):

$$
\left.T^{i_{1} \ldots i_{r}} \frac{\partial^{k}}{\partial v^{i_{1}} \ldots \partial v^{i_{r}}} D(x, v)\right|_{v=0}=\left.\kappa^{-1} \tilde{T}^{\mu_{1} \ldots \mu_{r}} \frac{\partial^{k}}{\partial \tilde{v}_{\mu_{1}} \ldots \partial \tilde{v}_{\mu_{r}}} \tilde{D}(\tilde{x}, \tilde{v})\right|_{v=0}
$$

for any invariant horizontal function $T^{i_{1} \ldots i_{r}} p_{i_{1}} \ldots p_{i_{r}}$ corresponding to $\tilde{T}^{\mu_{1}} \ldots \mu_{r} \tilde{p}_{\mu_{1}} \ldots \tilde{p}_{\mu_{r}}$ on $T^{*} Q$. Here we used the fact that any operator of the form $\left.f \mapsto \frac{\partial^{k}}{\partial v^{i_{1}} \ldots \partial v^{i_{r}}} f\right|_{v=0}$ may be obtained as a linear combination of operators of the form $\left.f \mapsto \frac{d^{k}}{d \lambda^{k}} f(\lambda a)\right|_{\lambda=0}$ for suitable vectors $a$. By Lemmas 1 and 3 we conclude:

$$
\left\langle\chi_{D} U \tilde{\phi}, \mathscr{Q}\left(T^{i_{1} \ldots i_{r}} p_{i_{1}} \cdots p_{i_{r}}\right) U \tilde{\psi}\right\rangle=C_{D}\left\langle\tilde{\phi}, \mathscr{Z}\left(\tilde{T}^{\mu_{1} \ldots \mu_{r}} \tilde{p}_{\mu_{1}} \ldots \tilde{p}_{\mu_{r}}\right) \bar{\psi}\right\rangle
$$

for any cylinder-shaped subset $D=\Phi^{-1}(\hat{V} \times W)$ and any $\tilde{\phi}, \tilde{\psi}$ with support contained in $\hat{V}$.

As the metric is $\mathbf{G}$-invariant, invariant functions are quantized by invariant operators. Hence, we get from (10):

$$
U^{-1} \mathscr{Q}\left(T^{i_{1} \ldots i_{r}} p_{i_{1}} \ldots p_{i_{r}}\right) U=\mathscr{Q}\left(\tilde{T}^{\mu_{1} \ldots \mu_{r}} \tilde{p}_{\mu_{1}} \ldots \tilde{p}_{\mu_{r}}\right) .
$$

Theorem 5. A necessary condition for the Dirac equivalence condition for strongly admissible quadratic observables to be fulfilled is that the orbits are totally geodesic submanifolds of $P$. In this case, the Dirac equivalence condition is satisfied for strongly admissible polynomials of arbitrary order.

Proof. We choose a local trivialisation of $E$ and adapted coordinates $\left(x_{i}\right)=\left(\phi_{\alpha}, \tilde{x}_{\mu}\right)$. For any G-invariant function $\rho(x)$ and any vertical quadratic polynomial, i.e., any polynomial of the form $f=T^{i j}(x) p_{i} p_{j}$ with

$$
T^{\alpha \mu}=T^{\mu \alpha}=T^{\mu \nu}=0
$$

for all $\mu, v, \alpha$, Eq. (7) yields:

$$
\mathscr{2}\left(\rho T^{i j} p_{i} p_{j}\right)=\rho \mathscr{Q}\left(T^{i j} p_{i} p_{j}\right)-T^{i j}\left(\nabla_{i} \rho\right) \nabla_{j}-\frac{1}{4}\left(T^{i j}\left(\nabla_{i} \nabla_{j} \rho\right)+2\left(\nabla_{i} \rho\right)\left(\nabla_{j} T^{i j}\right)\right) \text {. }
$$

If $T$ is an invariant tensor field, then $f$ and $\rho f$ are strongly admissible polynomials corresponding to the zero function on $T^{*} Q$. Hence, $\mathscr{Z}(f)$ and $\mathscr{Z}(\rho f)$ have to vanish on $\Omega_{\mathrm{phys}}^{1 / 2}(E)$, which gives the necessary condition:

$$
T^{i j}\left(\nabla_{i} \nabla_{j} \rho\right)+2\left(\nabla_{i} \rho\right)\left(\nabla_{j} T^{i j}\right)=0 .
$$

Now, even if $T$ is not invariant, the invariance of the function $\rho$ and the metric g guarantee that $\left(\nabla_{i} \nabla_{j} \rho\right)$ and $\left(\nabla_{i} \rho\right)$ are invariant tensor fields. Hence, as $\Phi_{g}$ is an affine transformation for any $g \in \mathbf{G}$ by the invariance of the metric $\mathbf{g}$ :

$\left(\left(\Phi_{g}\right)_{*} T\right)^{i j}\left(\nabla_{i} \nabla_{j} \rho\right)+2\left(\nabla_{i} \rho\right)\left(\nabla_{j}\left(\left(\Phi_{g}\right)_{*} T\right)^{i j}\right)=\left(\Phi_{g}\right)_{*}\left(T^{i j}\left(\nabla_{i} \nabla_{j} \rho\right)+2\left(\nabla_{i} \rho\right)\left(\nabla_{j} T^{i j}\right)\right)$ 
Introducing the Christoffel symbols $\Gamma_{j k}^{i}$, a direct computation using the invariance of $\rho$ and the verticality condition (11) yields:

$$
T^{i j}\left(\nabla_{i} \nabla_{j} \rho\right)+2\left(\nabla_{i} \rho\right)\left(\nabla_{j} T^{i j}\right)=T^{\alpha \beta} \Gamma_{\alpha \beta}^{\mu} \partial_{\mu} \rho
$$

If for an arbitrary choice of the $T^{\alpha \beta}$ in a fixed point there was a continuation to a G-invariant tensor field we could immediately conclude from (12) that $\Gamma_{\alpha \beta}^{\mu}$ in that arbitrary point was zero, and hence the orbits were totally geodesic submanifolds.

Now, for a non-free action a choice of $T^{\alpha \beta}(q)$ in a fixed point $q \in E$ only admits a $\mathbf{G}$-invariant continuation if it is invariant under the isotropy group $\mathbf{G}_{q}$. However, as the group action preserves verticality, for an arbitrary $T^{\alpha \beta}(q)$ the tensor

$$
\hat{T}(q)=\int\left(\phi_{g}\right)_{*} T(q) d \mu_{\mathbf{G}_{q}}
$$

is $\mathbf{G}_{q}$-invariant and vertical. Hence, (12), (14), and (13), and the fact that for any $g \in \mathbf{G}_{q}$ and $f \in \mathscr{F}(E)\left(\Phi_{g}\right)_{*} f(q)=f(q)$ imply:

$$
0=\hat{T}^{\alpha \beta}(q) \Gamma_{\alpha \beta}^{\mu}(q) \partial_{\mu} \rho=\operatorname{vol}\left(\mathbf{G}_{q}\right) T^{\alpha \beta}(q) \Gamma_{\alpha \beta}^{\mu}(q) \partial_{\mu} \rho,
$$

where $\operatorname{vol}\left(\mathbf{G}_{q}\right)$ denotes the volume of the isotropy group $\mathbf{G}_{q}$ with respect to the Haar measure $d \mu_{\mathbf{G}_{q}}$. (It is finite, as any isotropy group is compact by the properness of the action.) Thus, $\rho$ being an arbitrary $\mathbf{G}$-invariant function, $\Gamma_{\alpha \beta}^{\mu}(q)=0$ for any $q \in E$, and the orbits have to be totally geodesic submanifolds.

Using Theorem 4 we still have to prove that, provided the orbits are totally geodesic submanifolds, any vertical invariant polynomial is quantized by an operator vanishing on $\left(\mathscr{F}^{\mathbb{C}}(E)\right)_{\mathrm{G}}$. However, this may be easily shown using the formula (written in the notation of the preceding proof):

$$
\left.\frac{d^{k}}{d \lambda^{k}} D(x, \lambda a)\right|_{\lambda=0}=0 \quad \forall a \in \mathscr{V}_{x}=T_{x}(\mathbf{G} \cdot x)
$$

which follows from the invariance of the elements of $\left(\mathscr{F}^{\mathbb{C}}(E)\right)_{\mathrm{G}}$ along the orbits and the fact that vertical geodesics do not leave the orbits by definition of totally geodesic submanifolds. With Lemma 3 the vanishing of the operators corresponding to a vertical invariant polynomial on $\left(\mathscr{F}^{\mathbb{C}}(E)\right)_{\mathrm{G}}$ follows. Q.E.D.

Theorem 6. The Dirac equivalence condition for arbitrary invariant quadratic observables is fulfilled iff the orbits are totally geodesic submanifolds of $P$ and the connection form $\alpha$ on the $\mathbf{N} / \mathbf{G}$ principal bundle $P$ induced by the metric $\mathbf{g}$ on $E$ is flat. Then it is satisfied for invariant polynomials of arbitrary order. In particular, if $Q$ is simply connected, $E$ admits a metric such that the Dirac equivalence condition is fulfilled if and only if $P$ and hence $E$ are trivial bundles over $Q: P \cong Q \times \mathbf{N} / \mathbf{G}$, $E \cong Q \times \mathbf{G} / \mathbf{I}$.

Proof. Let the Dirac equivalence condition for arbitrary invariant quadratic functions on $T^{*} E$ be satisfied. Then, by the preceding theorem, the orbits are totally geodesic submanifolds. For an arbitrary point $q \in P \subset E$ any tangent vector to $P$, so in particular the fundamental vector $A^{*}(q)$ to an arbitrary $A \in \mathscr{N}$, is I-invariant and hence has a continuation to a G-invariant vertical vector field $\eta$. Choosing any horizontal invariant vector field $\tilde{X}^{h}$, the function $f=T^{i j} p_{i} p_{j}$ for $T=\eta \vee \tilde{X}^{h}$ is an invariant quadratic polynomial corresponding to the zero function on $T^{*} Q$, and 
hence has to be quantized by an operator vanishing on $\left(\mathscr{F}^{\mathbb{C}}(E)\right)_{\mathrm{G}}$. As in the free case a direct computation, using (7), yields the condition $\operatorname{div}\left(\nabla_{\eta} \tilde{X}^{h}\right)=0$. Using the fact that $g \eta$ is again an invariant vector field for any G-invariant function $g$, we conclude from the vanishing of the corresponding operators on the physical states that $\nabla_{\eta} \tilde{X}^{h}$ has to be vertical for any $\eta$, yielding, as in the free case:

$$
0=2 g\left(\nabla_{\eta} \tilde{X}^{h}, \tilde{Y}^{h}\right)=g\left(\left[\tilde{Y}^{h}, \tilde{X}^{h}\right], \eta\right)
$$

for all $\tilde{Y}, \tilde{X} \in \mathscr{X}(Q)$.

Now, the horizontal vector fields at any point in $P$ are tangent to $P$. Hence, $\left[\tilde{Y}^{h}, \tilde{X}^{h}\right]$ is tangent to $P$ when restricted to $P$. By its orthogonality to any fundamental vector field $A *$ for $A \in \mathscr{N}$ it has to be horizontal. Thus, we have shown that the horizontal distribution must be integrable and hence, the connection on $P$ has to be flat.

If $Q$ is simply connected, $P$ has to be a trivial principal bundle, and hence, $E$ has to be a trivial bundle as well.

Now assume that the orbits are totally geodesic and $\alpha$ is flat. As in this case any geodesic on $E$ projects to a geodesic on $Q$ that only depends on the projection of the starting velocity and the starting point we get:

$$
D(x, \lambda a)=D(x, \lambda \mathscr{H}(a)) .
$$

Using again the fact that any operator of the form $\left.f \mapsto \frac{\partial^{k}}{\partial v^{i_{1}} \ldots \partial v^{i_{r}}} f\right|_{v=0}$ may be obtained as a linear combination of operators of the form $\left.f \mapsto \frac{d^{k}}{d \lambda^{k}} f(\lambda a)\right|_{\lambda=0}$ for suitable vectors $a$, we may conclude that $\mathscr{2}\left(T^{i_{1}} \ldots i_{m}(x) p_{i_{1}} \ldots p_{i_{m}}\right)$ only depends on the horizontal part of $T$. Hence, using Theorem 4, Theorem 6 is proven. Q.E.D.

\section{Appendix: Proofs of Lemmas 2, 3}

Proof of Lemma 2. $X$ may be uniquely decomposed into a vertical and a horizontal invariant vector field, so we may assume that $X$ is either horizontal or vertical.

We first show that $(\operatorname{div} X)(q)=0$ for any $q \in Q$ for any invariant vertical vector field $X$. Without loss of generality we may assume $q \in P$. Let $\mathscr{G}=\mathscr{I} \oplus \mathscr{M}$ be a reductive decomposition of $\mathscr{G}$ (which always exists since $\mathbf{I}$ is compact), and $\left(A_{i}\right)$ a basis of $\mathscr{M}$. Then, the fundamental vector fields $A_{i}^{*}$ form a basis of the tangent spaces to the orbits in an open neighbourhood of $P$ in $E$ and we may, without loss of generality, choose the basis $\left(A_{i}\right)$ such that $X(q)=A_{1}^{*}(q)$. As $\operatorname{div} A_{i}^{*}=0$ by the G-invariance of the metric,

$$
\operatorname{div}\left(f^{i} A_{i}^{*}\right)(q)=\left(A_{i}^{*} \cdot f^{i}\right)(q)=\left.\frac{d}{d t} f^{i}\left(\exp \left(t A_{i}\right) q\right)\right|_{t=0} .
$$

Now, using the transformation behaviour of fundamental vector fields:

$$
\left(\Phi_{g}\right)_{*} A_{i}^{*}=\left(\operatorname{Ad}_{g} A_{i}\right)^{*}
$$

and the invariance of $X$, we get:

$$
X(g q)=\left(\operatorname{Ad}_{g} A_{1}\right)^{*}(g q)=\left(\left(\operatorname{Ad}_{g}\right)_{1}^{j} A_{j}\right)^{*}(g q)
$$


and hence:

$$
(\operatorname{div} X)(q)=\left.\frac{d}{d t}\left(\operatorname{Ad}_{\exp \left(t A_{j}\right)}\right)_{1}^{j}\right|_{t=0}=\left(\operatorname{ad}_{A_{j}}\right)_{1}^{j}=-\left(a d_{A_{1}}\right)_{j}^{j}=-\operatorname{tr} \overline{\operatorname{ad}}_{A_{1}}=0 .
$$

Here, the last equality follows from condition (3), as $A_{1}$ must be in $\mathscr{N}$, the Lie-algebra of the normalizer $\mathbf{N}$ of $\mathbf{I}$, for $X$ to be even $\mathbf{I}$-invariant.

Now assume that $X$ is a horizontal invariant vector field, i.e., the horizontal lift of a vector field $\tilde{X}$ on $Q: X=\tilde{X}^{h}$. Choosing again, without loss of generality, a point $q \in P$ and a basis $A_{i}$ like above, we get by the Levi-Civita formula defining the connection $\nabla$ :

$$
2 \mathbf{g}\left(\nabla_{\mathrm{A}_{i}^{*}} \tilde{X}^{h}, A_{j}^{*}\right)=\tilde{X}^{h} \mathbf{g}\left(A_{i}^{*}, A_{j}^{*}\right),
$$

as all other terms vanish by the horizontality and invariance of $X$. Using

$$
\mathscr{H}\left(\nabla_{\tilde{Y}^{h}} \tilde{X}^{h}\right)=\left(\tilde{\nabla}_{\tilde{Y}} \tilde{X}\right)^{h}
$$

we get:

$$
\operatorname{div} \tilde{X}^{h}=\left(\tilde{X}^{h}\right)_{; i}^{i}=\pi^{*}(\operatorname{div} \tilde{X})+h^{i j} \tilde{X}^{h} h_{i j}
$$

where $h_{i j}=\mathbf{g}\left(A_{i}^{*}, A_{j}^{*}\right)$ and $\left(h^{i j}\right)$ is the inverse matrix of $\left(h_{i j}\right)$.

Now, $h^{i j} \tilde{X}^{h} h_{i j}=\operatorname{det}\left(\mathbf{g}\left(A_{i}^{*}, A_{j}^{*}\right)\right)^{-1} X^{h} \operatorname{det}\left(\mathbf{g}\left(A_{i}^{*}, A_{j}^{*}\right)\right)=\kappa^{-1} X^{h} \kappa=0$ by the constancy of $\kappa$.

Hence,

$$
\int_{D} \operatorname{div} X d \mu=\int_{D} \pi^{*}(\operatorname{div} \tilde{X}) d \mu \propto \int_{\hat{V}} \operatorname{div} \tilde{X} d \tilde{u}=0,
$$

where the final equality follows from the condition on the support of $\tilde{X}$. Q.E.D.

Proof of Lemma 3: Formula (5) is a priori only applicable to functions $\phi, \psi$ of rapid decrease. However, it is still applicable to $\psi \in\left(\mathscr{F} \mathbb{C}^{\mathbb{2}}(E)\right)_{\mathrm{G}}$ if $\phi$ is smooth with compact support. (We are only dealing with formal differential operators and formal selfadjointness; a rigorous functional analytical treatment would require the use of generalized states in a Gelfand triple.) For such $\phi$ the derivative $\left.\frac{\partial}{\partial v^{i}}\right|_{v=0}$ acting on $\phi$ may be expressed by covariant derivatives $\nabla_{i}$ (with additional curvature terms arising in general) and moved to $\psi$ via partial integration. Here, no boundary terms arise, since $\phi$ is smooth and has compact support.

On the contrary, for $\hat{\phi}=\chi_{U} \phi$ there will be boundary terms and formula (5) is not valid in general for $\langle\hat{\phi}, \mathscr{2}(f) \psi\rangle$ for arbitrary smooth $\phi$ and an arbitrary open set $U$ of finite volume. In the case of $D$ being cylinder-shaped and $\phi \in\left(\mathscr{F} \mathbb{C}_{(E))_{\mathbf{G}}}\right.$ however, all boundary terms may be easily seen to be of the form $\int_{D} \operatorname{div} X d \mu$ for some vector fields $X$, which are invariant due to the invariance of $\phi, \psi$ and the metric g. Hence, under the assumptions of the lemma, the boundary terms vanish by Lemma 2 and formula (5) holds. Q.E.D.

\section{References}

1. Besse, A.L.: Einstein manifold. Berlin-Heidelberg New York: Springer, 1987

2. Coquereaux, R., Jadczyk, A.: Riemannian Geometry, Fiber Bundles, Kaluza-Klein Theories and All That .... Singapore: World Scientific, 1988 
3. Emmrich, C.: Equivalence of extrinsic and intrinsic quantization for observables not preserving the vertical polarization. Commun. Math. Phys. this issue

4. Gotay, M.J.: Constraints, reduction, and quantization. J. Math. Phys. 27, 2051-2066 (1986)

5. Tuynman, G.M.: Reduction, quantization, and nonunimodular groups. J. Math. Phys. 31, 83-90 (1990)

6. Tuynman, G.M.: Quantization of first class constraints with structure functions. Lett. Math. Phys. 21, 205-213 (1991)

7. Underhill, J.: Quantization on a manifold with connection. J. Math. Phys. 19, 1932-1935 (1978)

Communicated by H. Araki 